\title{
SHEAVES THAT FAIL TO REPRESENT MATRIX RINGS
}

\author{
MANUEL L. REYES \\ Dedicated to Professor Tsit Yuen Lam on the occasion of his seventieth birthday.
}

\begin{abstract}
There are two fundamental obstructions to representing noncommutative rings via sheaves. First, there is no subcanonical coverage on the opposite of the category of rings that includes all covering families in the big Zariski site. Second, there is no contravariant functor $F$ from the category of rings to the category of ringed categories whose composite with the global sections functor is naturally isomorphic to the identity, such that $F$ restricts to the Zariski spectrum functor Spec on the category of commutative rings (in a compatible way with the natural isomorphism). Both of these no-go results are proved by restricting attention to matrix rings.
\end{abstract}

\section{INTRODUCTION}

Recent results from [10] and [1, 2] show that there are fundamental obstructions to the extension of the Zariski spectrum functor from commutative rings to noncommutative rings. Specifically, if $F$ is a contravariant functor from rings to sets (or topological spaces) whose restriction to commutative rings is the usual prime spectrum Spec, then $F\left(\mathbb{M}_{n}(\mathbb{C})\right)=\varnothing$ for $n \geq 3$; see [10. There are various constructions that allow one to study "spaces without points." For instance, locales formalize the properties of the lattice of open sets on a topological space, and toposes formalize the properties of the category of sheaves of sets on a topological space. Thus one might wish to dodge this obstruction by considering the prime spectrum as a locale or topos (i.e., as a "pointless space"). But the same type of obstruction was shown to hold for functors taking values in the categories of locales and toposes; see [2].

(As the intended audience of this paper includes ring theorists, who are not necessarily acquainted with some of the categorical terminology used here, later sections include very basic and brief accounts of relevant topics with suggestions for further reading.)

Yet another attempt to avoid such obstructions would be to replace the spectrum of a commutative ring $R$ with certain sheaves associated to it. Of course, there is more than one kind of sheaf associated to a commutative ring $R$ and its spectrum. We focus on two specific types:

(I) The functor $\operatorname{Hom}_{\mathrm{cRing}}(-, R): \mathrm{cRing}^{\mathrm{op}} \rightarrow$ Set is a sheaf on the big Zariski site.

(II) The spectrum of $R$ is equipped with its structure sheaf $\mathcal{O}_{\mathrm{Spec}(R)}$, a particular member of the category of sheaves of rings on its underlying topological space.

By replacing $\operatorname{Spec}(R)$ with either of the objects in (I) or (II) above, one passes to a purely categorical setting that avoids any need for "points" or "open sets." One might hope that

Date: November 22, 2013.

2010 Mathematics Subject Classification. 14A22, 16B50, 18B25, 18F20.

Key words and phrases. noncommutative ring, Zariski spectrum, sheaf, coverage, ringed topos. 
after the spectrum of a ring is recast in either of these perspectives, it would easily extend to noncommutative rings. But to the contrary, the main results of this paper provide obstructions to noncommutative extensions of the spectrum in the vein of (I) and (II) above.

We now summarize the two major results to be proved below. The first obstruction concerns the view of $\operatorname{Spec}(R)$ expressed in $(\mathrm{I})$ above, and will be proved in Section 3 . A coverage on a category $\mathcal{C}$ is a designation of certain sets of morphisms in $\mathcal{C}$ as "covering families" and allows one to view certain contravariant set-valued functors on that category as sheaves. A coverage $J$ on a category $\mathcal{C}$ is called subcanonical if, for all $X \in \mathcal{C}$, the representable functor $\operatorname{Hom}_{\mathcal{C}}(-, X): \mathcal{C}^{\text {op }} \rightarrow$ Set is a sheaf for $J$.

Theorem 1.1. There is no subcanonical coverage $J$ on Ring ${ }^{\text {op }}$ such that every covering family for the big Zariski site is a covering family for (Ring $\left.{ }^{\mathrm{op}}, J\right)$.

Now we state the second obstruction, which is related to the view of $\operatorname{Spec}(R)$ given in (II) above. A ringed category $(\mathcal{X}, \mathcal{O})$ is category $\mathcal{X}$ with finite products, equipped with a ring object $\mathcal{O}$ in $\mathcal{X}$. These form the objects of a category RingedCat, which is described in detail in Definition 4.6. The ring of global sections of a ringed category $(\mathcal{X}, \mathcal{O})$ is the ring $\operatorname{Hom}_{\mathcal{X}}\left(1_{\mathcal{X}}, \mathcal{O}_{\mathcal{X}}\right)$, where $1_{\mathcal{X}}$ is the terminal object of $\mathcal{X}$. Ringed categories form a broad generalization of ringed spaces [4, II.2].

Theorem 1.2. Let $F:$ Ring $^{\mathrm{op}} \rightarrow$ RingedCat be a contravariant functor from the category of rings to the category of ringed categories, whose restriction to the category of commutative rings is isomorphic to Spec. Suppose that there exists a natural transformation of functors

$$
\eta: \operatorname{id}_{\text {Ring }} \rightarrow \Gamma \circ F
$$

(where $\Gamma:$ RingedCat ${ }^{\mathrm{op}} \rightarrow$ Ring is the global sections functor), which restricts on $\mathrm{cRing}$ to the canonical isomorphism from a commutative ring to the ring of global sections of its Zariski spectrum. Then for any ring $k$ and integer $n \geq 2$, the ringed category $F\left(\mathbb{M}_{n}(k)\right)$ has the zero structure ring object (and therefore has zero global sections). In particular, $\eta$ cannot be a natural isomorphism.

This result is proved in Section 4, A related obstruction was proved in [2]; see Remark 4.8 for a more detailed comparison of that result with the one above.

Theorems 1.1 and 1.2 actually follow from more general (but also more technical) results stated in each section.

Acknowledgments. It is my pleasure to thank Benno van den Berg, Luisa Fiorot, Chris Heunen, and Matthew Satriano for helpful discussions, suggestions, and comments.

Conventions. In this paper, all rings and ring homomorphisms are unital. The categories of sets, rings, and commutative rings are respectively denoted by Set, Ring, and cRing. We view a contravariant functor from a category $\mathcal{C}$ to a category $\mathcal{D}$ equivalently as an arrowreversing functor $\mathcal{C} \rightarrow \mathcal{D}$ or as an arrow-preserving functor $\mathcal{C}^{\mathrm{op}} \rightarrow \mathcal{D}$. We view all terminal objects of a category $\mathcal{C}$, which are canonically isomorphic, as being "equal" for the sake of simplicity. 


\section{A DIAGRAM OF RINGS}

The major results of this paper reduce to a basic diagram in the category of rings.

We introduce some notation to be used in the remainder of this paper. Let $k$ be a ring and $n \geq 1$ an integer. We let $k^{n}=\prod_{i=1}^{n} k$ denote the $n$-fold product. For $0 \leq i \leq n$ let $\pi_{i}: k^{n} \rightarrow k$ denote the projection map onto the $i$ th coordinate. In the ring $k^{n}$, let $e_{i}$ denote element whose $i$ th entry is 1 and whose other entries are zero. In the matrix ring $\mathbb{M}_{n}(k)$ we let $E_{i j}$ denote the matrix whose $(i, j)$-entry is 1 and whose other entries are zero. Also, we let $d: k^{n} \rightarrow \mathbb{M}_{n}(k)$ denote the usual diagonal embedding: the $k$-linear map sending $e_{i} \mapsto E_{i i}$.

Lemma 2.1. Let $k$ be a ring, and let $n \geq 2$ and $1 \leq i \leq n$ be integers. With notation as above, the diagram

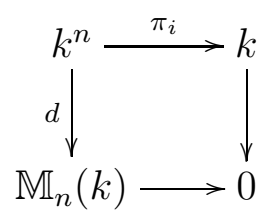

is a pushout in the category of rings.

Proof. Fix an integer $\ell \neq i$ with $1 \leq \ell \leq n$. Suppose that $R$ is a ring with homomorphisms $f: \mathbb{M}_{n}(k) \rightarrow R$ and $g: k \rightarrow R$ such that $f d=g \pi_{i}$. In the ring $\mathbb{M}_{n}(k)$, we have $1=$ $\sum_{j=1}^{n} E_{j \ell} E_{\ell \ell} E_{\ell j}$, so that $d\left(e_{\ell}\right)=E_{\ell \ell}$ generates the unit ideal. Applying $f$, it follows that $f d\left(e_{\ell}\right)$ generates the unit ideal in $R$. On the other hand $\pi_{i}\left(e_{\ell}\right)=0$, so that $g \pi_{i}\left(e_{\ell}\right)=0$. Now the image of $e_{\ell}$ in $R$ both generates the unit ideal and is zero, so $R=0$ and the diagram above is a pushout.

We will be concerned with the pullback diagram in Ring ${ }^{\text {op }}$ that is opposite to the diagram in Lemma 2.1. Let us speak intuitively about this "picture" in the case where $k=\mathbb{C}$ and $n=2$. For the moment, we will write $\operatorname{Spec}\left(\mathbb{M}_{2}(\mathbb{C})\right)$ for an imaginary geometric object corresponding to the matrix ring $\mathbb{M}_{2}(\mathbb{C})$. The pullback diagram is the following:

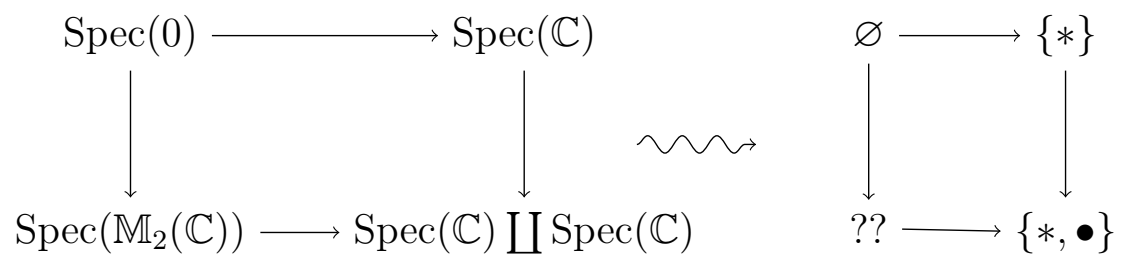

The maps $\operatorname{Spec}(\mathbb{C}) \rightarrow \operatorname{Spec}\left(\mathbb{C}^{2}\right)=\operatorname{Spec}(\mathbb{C}) \coprod \operatorname{Spec}(\mathbb{C})$ send the single point to one of two points. Traditionally, the pullback diagram above gives the fiber of the "space" $\operatorname{Spec}\left(\mathbb{M}_{2}(\mathbb{C})\right)$ over either point. Lemma 2.1 implies that both of these fibers are empty. In other words, we can imagine that $\operatorname{Spec}\left(\mathbb{M}_{2}(\mathbb{C})\right)$ maps to the two-point space $\operatorname{Spec}\left(\mathbb{C}^{2}\right)$, "without hitting either point."

Of course, the intuitive discussion above does not constitute rigorous mathematics. But the remainder of this paper can be viewed as providing two different ways to turn these ideas into precise results that obstruct certain approaches to realizing the category Ring ${ }^{\text {op }}$ as a category of "spaces." 
The following remark, related to the triviality of the pushout of diagram above, was kindly communicated to us by Luisa Fiorot: fibered coproducts in the category cRing (which are given by tensor products) commute with finite products, but Lemma 2.1 shows that fibered coproducts in the category Ring ("amalgamated free products") do not similarly commute with products. A noncommutative algebraist may see in this the difference between idempotents in commutative versus noncommutative rings: an idempotent in a commutative ring "neatly splits" the ring into a direct product of two commutative rings, while a noncentral idempotent in a noncommutative ring leads only to a Pierce corner decomposition which is not a direct product of rings.

\section{The FIRST OBSTRUCtion: SHEAVES ON A SITE}

We refer the reader to [5, C.2.1] for a thorough account of the theory of coverages and sheaves.

Let $\mathcal{C}$ be a category with pullbacks. A coverage $J$ on $\mathcal{C}$ is a rule assigning to every object $X \in \mathcal{C}$ a collection $J(X)$ of sets of morphisms in $\mathcal{C}$ with codomain $X$ (called "covering families") subject to the property:

- If $\left\{f_{i}: U_{i} \rightarrow X\right\} \in J(X)$ is a covering family and $g: Y \rightarrow X$ is a morphism in $\mathcal{C}$, then the family of pullbacks $\left\{g \times_{X} f_{i}: Y \times_{X} U_{i} \rightarrow Y\right\}$ is a covering family.

A site is a pair $(\mathcal{C}, J)$ where $\mathcal{C}$ is a category and $J$ is a coverage on $\mathcal{C}$.

(In the literature, a coverage is often defined by a weaker condition that can be stated even if $\mathcal{C}$ does not have pullbacks [5, Definition C.2.1.1]. However, for categories with pullbacks such as $\mathrm{cRing}^{\text {op }}$ or $\operatorname{Ring}^{\text {op }}$, the sheaves on a site $(\mathcal{C}, J)$ remain unchanged if $J$ is enlarged to be "stable under pullback" in the sense above; this follows from [5, Lemma C.2.1.6(i)].)

Example 3.1. The relevant example for us is the big Zariski site (see [8, VIII.6] and [11, Ex. 2.30]). This is the site (cRing ${ }^{\mathrm{op}}, J$ ) where for a commutative ring $R \in \mathrm{cRing}^{\mathrm{op}}$, the family $J(R)$ consists (up to isomorphism) of all families opposite to those of the form

$$
\left\{R \rightarrow R\left[r_{i}^{-1}\right] \mid r_{1}, \ldots, r_{n} \in R \text { and } \sum r_{i} R=R\right\} .
$$

(Stated geometrically, these are the collections of open immersions onto distinguished open subschemes of $\operatorname{Spec}(R)$ which collectively cover the space.) These families are stable under pullback in cRing $^{\text {op }}$ because, for any homomorphism $g: R \rightarrow S$ in cRing, the corresponding pushout diagram in $\mathrm{cRing}$ is

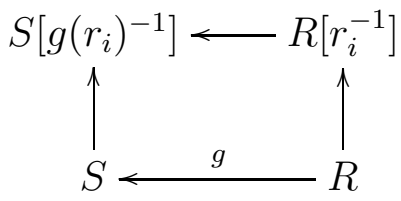

where $\sum r_{i} R=R$ implies that $\sum g\left(r_{i}\right) S=S$. We will refer to this coverage as the Zariski coverage.

For another example, a very special form of coverage used in algebraic geometry is a Grothendieck topology; see [8, Ch. III] and [11. While we will not recall the definition, a Grothendieck topology is a coverage that is closed under certain saturation conditions. Each 
coverage "generates" a Grothendieck topology that has the same sheaves as the original coverage; see [5, Proposition C.2.1.9].

The primary purpose of a coverage is to define sheaves on the corresponding site. A sheaf on a site $(\mathcal{C}, J)$ is a functor $F: \mathcal{C}^{\text {op }} \rightarrow$ Set such that, for every $X \in \mathcal{C}$ and every covering family $\left\{U_{i} \rightarrow X\right\} \in J(X)$, the following diagram of sets is an equalizer for all $i$ and $j$ :

$$
F(X) \rightarrow \prod_{i} F\left(U_{i}\right) \underset{F\left(p_{2}\right)}{\stackrel{F\left(p_{1}\right)}{\rightrightarrows}} F\left(U_{i} \times_{X} U_{j}\right),
$$

where $p_{1}: U_{i} \times_{X} U_{j} \rightarrow U_{i}$ and $p_{2}: U_{i} \times_{X} U_{j} \rightarrow U_{j}$ are the two "projections" from the pullbacks.

Recall that a coverage $J$ on a category $\mathcal{C}$ is called subcanonical if, for all $X \in \mathcal{C}$, the representable functor $\operatorname{Hom}_{\mathcal{C}}(-, X): \mathcal{C}^{\text {op }} \rightarrow$ Set is a sheaf for $J$. The important example for us is that the Zariski coverage on $\mathrm{cRing}^{\mathrm{op}}$ is subcanonical [11, 2.3.6].

We arrive at the first major result. It states that any coverage on Ring ${ }^{\text {op }}$ which mildly attempts to extend the Zariski coverage on $\mathrm{cRing}^{\mathrm{op}}$ will fail to distinguish matrix rings from the zero ring.

We denote the pushout (or fibered coproduct) of two ring homomorphisms $R \rightarrow S$ and $R \rightarrow T$ by $S *_{R} T$. This is a sort of "amalgamated free product" of $S$ and $T$ relative to $R$. If $k$ is a ring, then a ring over $k$ is a ring $R$ equipped with a homomorphism $k \rightarrow R$.

Theorem 3.2. Let $k$ be a ring and $n \geq 2$ be an integer. Let $J$ be a coverage on the category Ring ${ }^{\text {op }}$ for which the opposite of the family $\left\{\pi_{i}: k^{n} \rightarrow k \mid i=1, \ldots, n\right\}$ is a covering family. Then for every ring $R$ over $k$, the opposite of the singleton family $\left\{\mathbb{M}_{n}(R) \rightarrow 0\right\}$ is a covering family for $J$, and for any sheaf $F$ on the site (Ring ${ }^{\mathrm{op}}, J$ ), this morphism induces an isomorphism of sets $F\left(\mathbb{M}_{n}(R)\right) \cong F(0)$.

Proof. By the definition of a coverage, the pullbacks in Ring ${ }^{\text {op }}$ (i.e., the pushouts in Ring) of the maps $\pi_{i}$ along the diagonal map $k^{n} \rightarrow \mathbb{M}_{n}(k)$ must form a covering family for $J$. But by Lemma 2.1, these pushouts are all zero maps $\mathbb{M}_{n}(k) \rightarrow 0$. Thus the opposite family of $\left\{\mathbb{M}_{n}(k) \rightarrow 0\right\}$ is a covering family. The homomorphism $k \rightarrow R$ induces a homomorphism $g: \mathbb{M}_{n}(k) \rightarrow \mathbb{M}_{n}(R)$, and of course the pushout of the morphism $\mathbb{M}_{n}(k) \rightarrow 0$ along $g$ is $\mathbb{M}_{n}(R) \rightarrow 0$. As this is a pullback in Ring ${ }^{\text {op }}$, the coverage axiom implies that $\left\{\mathbb{M}_{n}(R) \rightarrow 0\right\}$ is a covering family for $J$.

Let $F$ be a sheaf on $\left(\operatorname{Ring}^{\text {op }}, J\right)$; in particular, $F$ is a functor Ring $=\left(\operatorname{Ring}^{\text {op }}\right)^{\text {op }} \rightarrow$ Set. The fibered coproduct $0 *_{S} 0$ in Ring for any ring $S$ (relative to the unique homomorphism $S \rightarrow 0$ ) is easily seen to be zero. Now the sheaf axiom declares that the following diagram must be an equalizer:

$$
F\left(\mathbb{M}_{n}(R)\right) \rightarrow F(0) \rightrightarrows F\left(0 *_{\mathbb{M}_{n}(R)} 0\right)=F(0) .
$$

The two arrows $F(0) \rightarrow F(0)$ are equal because they are both the image under $F$ of the unique ring homomorphism $0 \rightarrow 0$. Thus the equalizer of these arrows is $F(0)$ itself, meaning that $F\left(\mathbb{M}_{n}(R)\right) \cong F(0)$.

Suppose that $(\mathcal{C}, J)$ and $(\mathcal{D}, K)$ are sites and that $F: C \rightarrow D$ is a functor. We say that $F$ preserves covering families if, for every covering family $\left\{f_{i}: U_{i} \rightarrow X\right\}$ for $(\mathcal{C}, J)$, the set $\left\{F\left(f_{i}\right): F\left(U_{i}\right) \rightarrow F(X)\right\}$ is a covering family for $(\mathcal{D}, K)$. 
Corollary 3.3. There is no coverage $J$ on Ring $^{\text {op }}$ such that the functor

$$
F=\operatorname{Hom}_{\text {Ring }}^{\mathrm{op}}(-, 0)=\operatorname{Hom}_{\text {Ring }}(0,-): \operatorname{Ring}^{\text {op }} \rightarrow \text { Set }
$$

is a sheaf on $\left(\operatorname{Ring}^{\mathrm{op}}, J\right)$ and the inclusion $\mathrm{cRing}^{\mathrm{op}} \hookrightarrow \mathrm{Ring}^{\mathrm{op}}$ preserves Zariski covering families.

Proof. Assume for contradiction that such a subcanonical coverage $J$ exists. For any field $k$, the projections $\pi_{i}: k \times k \rightarrow k(i=1,2)$ together form a covering family on the big Zariski site; on the level of schemes, these morphisms correspond to the open immersions $\operatorname{Spec}(k) \rightarrow \operatorname{Spec}(k \times k)=\operatorname{Spec}(k) \coprod \operatorname{Spec}(k)$ mapping the unique point of $\operatorname{Spec}(k)$ onto either of the two points of $\operatorname{Spec}(k \times k)$. By hypothesis, this family must also cover $k \times k$ in the topology $J$ on Ring ${ }^{\text {op }}$. So by Theorem [3.2, the sheaf $F$ assigns isomorphic sets to 0 and $\mathbb{M}_{2}(k)$. But this contradicts the fact that $\operatorname{Hom}_{\text {Ring }}(0,0)$ is a singleton and $\operatorname{Hom}_{\text {Ring }}\left(0, \mathbb{M}_{2}(k)\right)$ is empty.

(One could prove slightly stronger statements of Theorem 3.2 and Corollary 3.3 which assume only that the coverage $J$ on Ring ${ }^{\text {op }}$ has a covering family which "refines" the family $\left\{\pi_{i}: k^{n} \rightarrow k\right\}$, or respectively that $J$ refines all Zariski covering families. This would make use of [5, Lemma C.2.1.6(i)]. We have fixed our definitions and stated our results in order to keep the exposition as self-contained as possible.)

We obtain Theorem 1.1 as a special case of the preceding result.

Proof of Theorem 1.1. If $J$ is a subcanonical coverage on Ring ${ }^{\text {op }}$, then the functor

$$
\operatorname{Hom}_{\text {Ring }}{ }^{\text {op }}(-, 0): \text { Ring }^{\text {op }} \rightarrow \text { Set }
$$

is a sheaf for $\left(\operatorname{Ring}^{\text {op }}, J\right)$. The claim immediately follows from Corollary 3.3 .

The results proved above do not indicate that every attempt to "do geometry" with the category Ring ${ }^{\text {op }}$ is futile. To the contrary, such approaches to noncommutative geometry have been developed in [7] and [9]. These approaches all happen to work with analogues (not generalizations) of phenomena from commutative algebraic geometry, and deal with presheaves of rings on the category Ring ${ }^{\text {op }}$. The results presented here simply show that such indirect approaches are necessary.

As each scheme $X$ defines a sheaf $\operatorname{Hom}(\operatorname{Spec}(-), X):\left(\text { cRing }^{\text {op }}\right)^{\text {op }} \rightarrow$ Set on the big Zariski site, we see that the obstruction in Theorem 1.1 expresses, at least in part, the difficulty in producing a notion of "gluing" of noncommutative spaces.

To close this section, we share an observation communicated to us by Benno van den Berg. It is possible to define sheaves even in the case when covering families are not stable under pullback (and therefore do not form a coverage in the traditional sense). For example, see the discussion surrounding [5, Example C.2.1.13]. The resulting categories of sheaves are generally much less well-behaved. We do not know whether the obstructions proved in this section persist or can be avoided if one works with such a generalized notion of sheaf on a category. 


\section{ThE SECOND OBSTRUCTION: SHEAVES OF RINGS}

To present the final no-go result, let us imagine the most utopian setting for noncommutative geometry. There should be a category of noncommutative topological spaces. (The need for such a category, extending the usual category of "commutative" topological spaces, is suggested by the no-go results presented in [10] and [2].) Given a noncommutative topological space $X$, one would wish to have some collection of sheaves on that space. Even in the event that there is some obstruction to general categories of "sheaves of sets on $X$ " in the fully noncommutative setting (see Question 4.9), one would at least hope that there is a suitable category $\operatorname{ShR}(X)$ of "sheaves of rings on $X$." Of course, there should be a global sections functor $\Gamma(X,-): \operatorname{ShR}(X) \rightarrow$ Ring. Given a morphism of noncommutative spaces $f: X \rightarrow Y$ and a sheaf of rings $\mathcal{O}_{X}$ on $X$, one would wish for a "direct image sheaf" $f_{*} \mathcal{O}_{Y}$ that is a sheaf of rings on $X$.

In case $f: X \rightarrow Y$ is a continuous function between honest ("commutative") topological spaces, the direct image sheaf $f_{*} \mathcal{O}_{X}$ is defined by $f_{*} \mathcal{O}_{X}(U)=\mathcal{O}_{X}\left(f^{-1}(U)\right)$ for each open set $U \subseteq Y$. Thus, by the very definition of the direct image, $\Gamma\left(Y, f_{*} \mathcal{O}_{X}\right)=\Gamma\left(X, \mathcal{O}_{X}\right)$. That is, direct images preserve global sections.

Remark 4.1. We will frequently refer to categories of "large" categories, whose morphisms are functors and therefore might allow for "large" hom-sets. This is safely handled using a "second-order universe" as described, for instance, in [3, §6.4]. To be explicit, assume a Grothendieck universe $U_{1}$ and let Set be the category of sets that are elements of $U_{1}$. Assume there is another universe $U_{2}$ such that $U_{1} \in U_{2}$. Define Cat to be the category of all categories $\mathcal{C}$ such that $\operatorname{Obj}(\mathcal{C}) \in U_{2}$, with functors for morphisms. (In particular, the category Set of "small" sets will be an object of Cat.) Then every "category of categories" we consider will be a subcategory of Cat. These technicalities do not pose a serious issue, and we will mostly ignore size considerations in the rest of this paper.

The following axioms express the minimal requirements for a category $\mathcal{C}$ to behave as a category of "ringed noncommutative spaces" for which morphisms have "direct images of sheaves of rings that preserve global sections."

Hypothesis 4.2. In this section, we will consider categories $\mathcal{C}$ satisfying the following properties:

(A) Every object $X \in \mathcal{C}$ is a 3 -tuple $X=\left(\operatorname{ShR}(X), \mathcal{O}_{X}, \Gamma(X,-)\right)$ such that:

(A1) $\operatorname{ShR}(X)$ is a category;

(A3) $\mathcal{O}_{X}$ is an object of $\operatorname{ShR}(X)$;

(A2) $\Gamma(X,-): \operatorname{ShR}(X) \rightarrow$ Ring is a functor.

(B) Every morphism $f: X \rightarrow Y$ in $\mathcal{C}$ is an ordered pair $f=\left(f_{*}, f\right)$ such that:

(B1) $f_{*}: \operatorname{ShR}(X) \rightarrow \operatorname{ShR}(Y)$ is a functor satisfying $\Gamma\left(Y, f_{*}\left(\mathcal{O}_{X}\right)\right) \cong \Gamma\left(X, \mathcal{O}_{X}\right)$;

(B2) $f: \mathcal{O}_{Y} \rightarrow f_{*} \mathcal{O}_{X}$ is a morphism in $\operatorname{ShR}(Y)$.

The prototypical example of a triple satisfying axiom $(\mathrm{A})$ is $\left(\operatorname{ShRing}(X), \mathcal{O}_{X}, \Gamma(X,-)\right)$ where $X$ is a topological space, $\operatorname{ShRing}(X)$ is the category of sheaves of rings on $X, \mathcal{O}_{X} \in$ $\operatorname{ShRing}(X)$ is a particular sheaf of rings, and $\Gamma(X,-): \operatorname{ShRing}(X) \rightarrow \operatorname{Ring}$ is the global sections functor. We will return to this example (in much broader generality) when we present the proof of Theorem 1.2 later in this section. 
In the following, we will view the composite of two functors $F: \mathcal{C}_{1}^{\text {op }} \rightarrow \mathcal{C}_{2}$ and $G: \mathcal{C}_{2}^{\text {op }} \rightarrow \mathcal{C}_{3}$ as a covariant functor $G \circ F: \mathcal{C}_{1} \rightarrow \mathcal{C}_{3}$.

Hypothesis 4.3. Let $\mathcal{C}$ be a category satisfying Hypothesis 4.2. We will consider functors $\mathrm{Sp}: \operatorname{Ring}^{\mathrm{op}} \rightarrow \mathcal{C}$ with the following properties:

(A) The restriction of Sp to cRing ${ }^{\text {op }}$ is the functor

$$
R \mapsto\left(\operatorname{ShRing}(\operatorname{Spec}(R)), \mathcal{O}_{\operatorname{Spec}(R)}, \Gamma(\operatorname{Spec}(R),-)\right) .
$$

(B) There is a natural transformation $\eta$ : $\operatorname{id}_{\operatorname{Ring}} \rightarrow \Gamma\left(\operatorname{Sp}(-), \mathcal{O}_{\mathrm{Sp}(-)}\right)$ of endofunctors of Ring whose restriction to cRing is the isomorphism of $\mathrm{id}_{\mathrm{cRing}}$ with $\Gamma\left(\operatorname{Spec}(-), \mathcal{O}_{\mathrm{Spec}(-)}\right)$. (That is, $\eta$ restricts to the unit of the adjunction between the Spec and global sections functors).

For convenience, the following lemma is separated out from the proof of Theorem 4.5. We continue to use the notation introduced in Section 2.

Lemma 4.4. Let $k$ be a commutative ring, and let $\mathcal{O}$ be a sheaf of rings on the topological space $\operatorname{Spec}\left(k^{n}\right)$ with a morphism of sheaves of rings $\mathcal{O}_{\text {Spec } k^{n}} \rightarrow \mathcal{O}$ such that the induced map on global sections factors through the diagonal morphism $d: k^{n} \hookrightarrow \mathbb{M}_{n}(k)$, as follows:

$$
\Gamma\left(\operatorname{Spec}\left(k^{n}\right), \mathcal{O}_{\operatorname{Spec}\left(k^{n}\right)}\right) \cong k^{n} \stackrel{d}{\longrightarrow} \mathbb{M}_{n}(k) \rightarrow \Gamma\left(\operatorname{Spec}\left(k^{n}\right), \mathcal{O}\right)
$$

Then $\mathcal{O}$ is the zero sheaf of rings.

Proof. Let $U_{j}$ be the clopen subset of $X=$ Spec $k^{n}$ whose embedding $U_{j} \hookrightarrow X$ corresponds to the projection $k^{n} \rightarrow k$ onto the $j$ th factor, so that $X=\coprod_{j=1}^{n} U_{j}$. Then for each $j$ the diagram

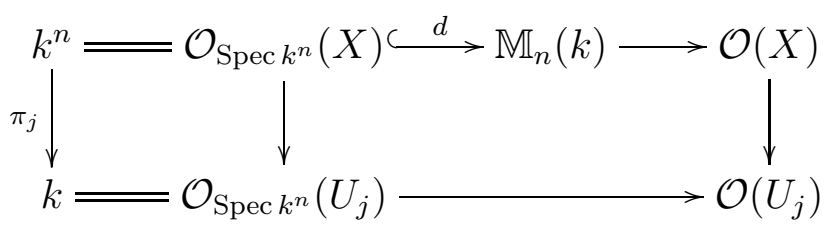

is commutative. It follows from Lemma 2.1 that $\mathcal{O}\left(U_{j}\right)=0$. Because the open sets $U_{j}$ cover $X$, it follows from the gluing axiom for sheaves that $\mathcal{O}(X)=0$. The fact that $\mathcal{O}$ is a sheaf of rings means that its restriction maps are ring homomorphisms. So for all open subsets $U$ of Spec $k^{n}$, the existence of a ring homomorphism $0=\mathcal{O}(X) \rightarrow \mathcal{O}(U)$ via restriction to $U$ implies that $\mathcal{O}(U)=0$. Hence $\mathcal{O}$ is the zero sheaf.

Theorem 4.5. Let $\mathcal{C}$ be a category satisfying Hypothesis 4.2 with a functor $\mathrm{Sp}: \mathrm{Ring}^{\mathrm{op}} \rightarrow \mathcal{C}$ satisfying Hypothesis 4.3. Let $k$ be any ring and $n \geq 2$ an integer. Then

$$
\Gamma\left(\operatorname{Sp}\left(\mathbb{M}_{n}(k)\right), \mathcal{O}_{\mathrm{Sp}\left(\mathbb{M}_{n}(k)\right)}\right)=0 .
$$

In particular, if $k$ is nonzero then $\Gamma\left(\operatorname{Sp}\left(\mathbb{M}_{n}(k)\right), \mathcal{O}_{\mathrm{Sp}\left(\mathbb{M}_{n}(k)\right)}\right) ¥ \mathbb{M}_{n}(k)$.

Proof. It suffices to consider the case $k=\mathbb{Z}$. For, given any ring $k$, the unique homomorphism $\mathbb{Z} \rightarrow k$ induces a homomorphism

$$
\Gamma\left(\operatorname{Sp}\left(\mathbb{M}_{n}(\mathbb{Z})\right), \mathcal{O}_{\mathrm{Sp}\left(\mathbb{M}_{n}(\mathbb{Z})\right)}\right) \rightarrow \Gamma\left(\operatorname{Sp}\left(\mathbb{M}_{n}(k)\right), \mathcal{O}_{\mathrm{Sp}\left(\mathbb{M}_{n}(k)\right)}\right) .
$$

If the ring on the left is zero, then the ring on the right must also be zero. 
Recall the notation for the diagonal morphism $d: \mathbb{Z}^{n} \hookrightarrow \mathbb{M}_{n}(\mathbb{Z})$. In the category $\mathcal{C}$, there is a resulting morphism $f=\operatorname{Sp}(d): \operatorname{Sp}\left(\mathbb{M}_{n}(\mathbb{Z})\right) \rightarrow \operatorname{Sp}\left(\mathbb{Z}^{n}\right)$. This morphism consists of a functor $f_{*}: \operatorname{ShR}\left(\operatorname{Sp}\left(\mathbb{M}_{n}(\mathbb{Z})\right)\right) \rightarrow \operatorname{ShR}\left(\operatorname{Sp}\left(\mathbb{Z}^{n}\right)\right)=\operatorname{ShRing}\left(\operatorname{Spec}\left(\mathbb{Z}^{n}\right)\right)$ along with a morphism $\underline{f}: \mathcal{O}_{\mathrm{Sp}\left(\mathbb{Z}^{n}\right)}=\mathcal{O}_{\operatorname{Spec}\left(\mathbb{Z}^{n}\right)} \rightarrow f_{*} \mathcal{O}_{\operatorname{Sp}\left(\mathbb{M}_{n}(\mathbb{Z})\right)}$ in the category $\operatorname{ShRing}\left(\operatorname{Spec}\left(\mathbb{Z}^{n}\right)\right)$.

Write $X=\operatorname{Sp}\left(\mathbb{M}_{n}(\mathbb{Z})\right)$. Property $(B)$ of Hypothesis 4.3 provides the following commutative diagram:

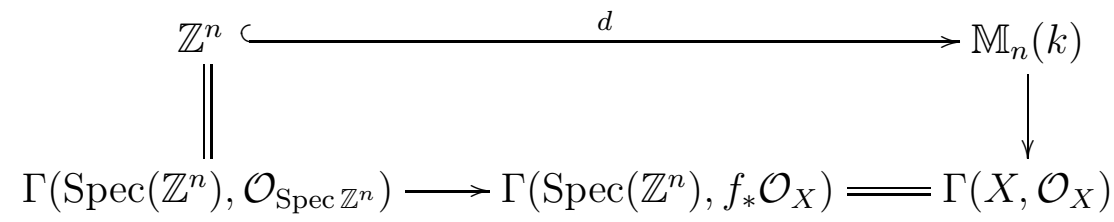

where the vertical arrows are the components of the natural transformation $\eta$, and the isomorphism $\mathbb{Z}^{n} \cong \Gamma\left(\operatorname{Spec}\left(\mathbb{Z}^{n}\right), \mathcal{O}_{\operatorname{Spec}\left(\mathbb{Z}^{n}\right)}\right)$ coincides with the one provided by the duality between commutative rings and affine schemes. It follows from Lemma 4.4 that $f_{*} \mathcal{O}_{\operatorname{Sp}\left(\mathbb{M}_{n}(\mathbb{Z})\right)}$ is the zero sheaf. From condition (B1) of Hypothesis 4.2 we conclude that

$$
\Gamma\left(\operatorname{Sp}\left(\mathbb{M}_{n}(\mathbb{Z})\right), \mathcal{O}_{\mathrm{Sp}\left(\mathbb{M}_{n}(\mathbb{Z})\right)}\right)=\Gamma\left(\operatorname{Spec}\left(\mathbb{Z}^{n}\right), f_{*} \mathcal{O}_{\mathrm{Sp}\left(\mathbb{M}_{n}(\mathbb{Z})\right)}\right)=0
$$

The "preservation of global sections" in Hypothesis 4.2(B1) plays a crucial role in the proof above. We do not know what can be said if that hypothesis is omitted or weakened (or similarly, if the analogous hypothesis on fpCat below is omitted). The latter part of Question 4.9 is related to this.

A rather general category satisfying Hypothesis 4.2 can be obtained from ringed categories, which we now proceed to define. Let $\mathcal{X}$ be a category with finite products. In particular, $\mathcal{X}$ has a terminal object $1_{\mathcal{X}}$, which is the product indexed by the empty set. We define the global sections functor $\Gamma(\mathcal{X},-): \mathcal{X} \rightarrow$ Set to be the hom-functor $\Gamma(\mathcal{X},-)=\operatorname{Hom}_{\mathcal{X}}\left(1_{\mathcal{X}},-\right)$. (This agrees with the usual global sections functor in case $\mathcal{X}$ is a topos, such as the category of sheaves on a topological space or locale [8, p 135].)

A ring object of $\mathcal{X}$ is an object $\mathcal{O} \in \mathcal{X}$ that is equipped with "zero," "unity," "addition," "subtraction," and "multiplication" morphisms

$$
0,1 \in \operatorname{Hom}_{\mathcal{X}}\left(1_{\mathcal{X}}, \mathcal{O}\right), \quad+, \bullet \in \operatorname{Hom}_{\mathcal{X}}(\mathcal{O} \times \mathcal{O}, \mathcal{O}), \quad \text { and } \quad-\in \operatorname{Hom}(\mathcal{O}, \mathcal{O}),
$$

such that the usual axioms for (associative, unital) rings hold when expressed as commuting diagrams in $\mathcal{X}$ between products of copies of $\mathcal{O}$ (see [8, VIII..5]). A morphism of ring objects $\mathcal{O}_{1}$ and $\mathcal{O}_{2}$ in $\mathcal{X}$ is a morphism $\mathcal{O}_{1} \rightarrow \mathcal{O}_{2}$ in $\mathcal{X}$ such that the induced diagrams relating the zero, unity, addition, subtraction, and multiplication of $\mathcal{O}_{1}$ and $\mathcal{O}_{2}$ are commutative.

If $\mathcal{X}$ is a category with finite products, we write $\operatorname{Ring} \operatorname{Obj}(\mathcal{X})$ for the category of ring objects in $\mathcal{X}$. The structure of a ring object $\mathcal{O} \in \operatorname{RingObj}(\mathcal{X})$ naturally induces a ring structure on the global sections $\operatorname{Hom}_{\mathcal{X}}(A, \mathcal{O})$ for any $A \in \mathcal{X}$ (essentially due to the fact that the functor $\operatorname{Hom}_{\mathcal{X}}(A,-): \mathcal{X} \rightarrow$ Set preserves products). In this way, the global sections functor $\Gamma(\mathcal{X},-)=\operatorname{Hom}_{\mathcal{X}}\left(1_{\mathcal{X}},-\right): \mathcal{X} \rightarrow$ Set induces a functor $\operatorname{RingObj}(\mathcal{X}) \rightarrow \operatorname{Ring}$, which we also denote by $\Gamma(\mathcal{X},-)$.

If $f_{*}: \mathcal{X} \rightarrow \mathcal{Y}$ is a functor between categories with finite products that preserves finite products, then $f_{*}$ induces a natural transformation $\Gamma(\mathcal{X},-) \rightarrow \Gamma\left(\mathcal{Y}, f_{*}(-)\right)$ of functors $\mathcal{X} \rightarrow$ 
Set, as follows. Because a terminal object is a product indexed by the empty set, we have $f^{*}\left(1_{\mathcal{X}}\right)=1_{\mathcal{Y}}$. Thus for any $A \in \mathcal{X}, f_{*}$ induces the arrow below:

$$
\Gamma(\mathcal{X}, A)=\operatorname{Hom}_{\mathcal{X}}\left(1_{\mathcal{X}}, A\right) \rightarrow \operatorname{Hom}_{\mathcal{Y}}\left(f_{*}\left(1_{\mathcal{X}}\right), f_{*}(A)\right)=\operatorname{Hom}_{\mathcal{Y}}\left(1_{\mathcal{Y}}, f_{*}(A)\right)=\Gamma\left(\mathcal{Y}, f_{*}(A)\right)
$$

We say that $f_{*}$ preserves global sections if the transformation above is a natural isomorphism $\Gamma(\mathcal{X},-) \cong \Gamma\left(\mathcal{Y}, f_{*}(-)\right)$. We let $\mathrm{fpCat}$ denote the category whose objects are categories with finite products and whose morphisms are functors that preserve finite products and global sections. (Recall Remark 4.1 regarding size issues.)

Definition 4.6. A ringed category is a pair $(\mathcal{X}, \mathcal{O})$ where $\mathcal{X}$ is a category with finite products and $\mathcal{O}$ is a ring object in $\mathcal{X}$; we refer to $\mathcal{O}$ as the structure ring object of $(\mathcal{X}, \mathcal{O})$. A morphism of ringed categories $\left(\mathcal{X}, \mathcal{O}_{\mathcal{X}}\right) \rightarrow\left(\mathcal{Y}, \mathcal{O}_{\mathcal{Y}}\right)$ is a pair $\left(f_{*}, f\right)$ where $f_{*}: \mathcal{X} \rightarrow \mathcal{Y}$ is a functor in fpCat and $f: \mathcal{O}_{\mathcal{Y}} \rightarrow f_{*} \mathcal{O}_{\mathcal{X}}$ is a morphism in RingObj( $\left.\mathcal{Y}\right)$. Given morphisms $\left(f_{*}, f\right):\left(\mathcal{X}, \mathcal{O}_{\mathcal{X}}\right) \rightarrow\left(\mathcal{Y}, \overline{\mathcal{O}}_{\mathcal{Y}}\right)$ and $\left(g_{*}, g\right):\left(\mathcal{Y}, \mathcal{O}_{Y}\right) \rightarrow\left(\mathcal{Z}, \mathcal{O}_{\mathcal{Z}}\right)$, we define their composite to be the morphism

$$
\left(g_{*}, \underline{g}\right) \circ\left(f_{*}, \underline{f}\right)=\left(g_{*} \circ f_{*}, \underline{g} \circ g_{*}(\underline{f})\right) .
$$

This operation is readily seen to be associative. The category RingedCat has ringed categories for objects, with morphisms and composition rule as above. The assignment $\left(\mathcal{X}, \mathcal{O}_{\mathcal{X}}\right) \mapsto$ $\Gamma\left(\mathcal{X}, \mathcal{O}_{\mathcal{X}}\right)$ defines a functor $\Gamma:$ RingedCat ${ }^{\text {op }} \rightarrow$ Ring, which we also refer to as the global sections functor.

The zero ring object of a category with finite products is the terminal object, equipped with its unique structure as a ring object. The following observation will prove useful.

Lemma 4.7. Let $(\mathcal{X}, \mathcal{O})$ be a ringed category. If $\Gamma(\mathcal{X}, \mathcal{O})$ is the zero ring, then $\mathcal{O}$ is the zero ring object on $\mathcal{X}$.

Proof. Because $\mathcal{O}$ is a ring object in $\mathcal{X}$, the $\operatorname{Hom}$-functor $\operatorname{Hom}_{\mathcal{X}}(-, \mathcal{O})$ has the extra structure of a functor $\mathcal{X}^{\text {op }} \rightarrow$ Ring. Given any object $A \in \mathcal{X}$, the morphism to the terminal object $A \rightarrow 1_{\mathcal{X}}$ induces a ring homomorphism

$$
0=\Gamma\left(\mathcal{X}, \mathcal{O}_{\mathcal{X}}\right)=\operatorname{Hom}_{\mathcal{X}}\left(1_{\mathcal{X}}, \mathcal{O}_{\mathcal{X}}\right) \rightarrow \operatorname{Hom}_{\mathcal{X}}(A, \mathcal{X})
$$

This implies that $\operatorname{Hom}_{\mathcal{X}}(A, \mathcal{O})$ is the zero ring and consequently is a singleton set for all $A$. Thus $\mathcal{O}$ is a terminal object, and therefore is a zero ring object.

For the category $\operatorname{Sh}(X)$ of sheaves of sets on a topological space $X$, the category of ring objects in $\operatorname{Sh}(X)$ is the same as the category of sheaves of rings on $X$; see [8, II.7]. This provides a functor from the category of ringed spaces [4, II.2] to the category of ringed categories, which is faithful. In this way, we view the category of ringed spaces as a subcategory of RingedCat. By composing with this embedding, we may consider the Zariski spectrum as a functor Spec: cRing $^{\text {op }} \rightarrow$ RingedCat.

We are finally prepared to prove the second of the major results of Section 1 .

Proof of Theorem 1.2. Let $\mathcal{C}$ be the category defined as follows:

- The objects of $\mathcal{C}$ are ordered triples $\left(\operatorname{RingObj}(\mathcal{X}), \mathcal{O}_{\mathcal{X}}, \Gamma(\mathcal{X},-)\right)$, where $\left(\mathcal{X}, \mathcal{O}_{\mathcal{X}}\right)$ is a ringed category and $\Gamma(\mathcal{X},-): \operatorname{RingObj}(\mathcal{X}) \rightarrow \operatorname{Ring}$ is the (co)restriction of the global sections functor on $\mathcal{X}$. 
- A morphism

$$
f:\left(\operatorname{RingObj}(\mathcal{X}), \mathcal{O}_{\mathcal{X}}, \Gamma(\mathcal{X},-)\right) \rightarrow\left(\operatorname{RingObj}(\mathcal{Y}), \mathcal{O}_{\mathcal{Y}}, \Gamma(\mathcal{Y},-)\right)
$$

is a morphism of ringed categories $f=\left(f_{*}, f\right):\left(\mathcal{X}, \mathcal{O}_{\mathcal{X}}\right) \rightarrow\left(\mathcal{Y}, \mathcal{O}_{\mathcal{Y}}\right)$.

The construction of RingedCat and the discussion preceding this proof indicate that $\mathcal{C}$ satisfies Hypothesis 4.2. There is a rather obvious functor $G$ : RingedCat $\rightarrow \mathcal{C}$, defined by $\left(\mathcal{X}, \mathcal{O}_{\mathcal{X}}\right) \mapsto$ $\left(\operatorname{Ring} \operatorname{Obj}(\mathcal{X}), \mathcal{O}_{\mathcal{X}}, \Gamma(\mathcal{X},-)\right)$ for objects and defined in the trivial way on morphisms.

Let $F:$ Ring $^{\mathrm{op}} \rightarrow$ RingedCat and $\eta:$ id $_{\text {Ring }} \rightarrow \Gamma \circ F$ be as in the statement of Theorem 1.2, and define $\mathrm{Sp}=G \circ F:$ Ring $^{\mathrm{op}} \rightarrow \mathcal{C}$. By construction of $G$, the functor

$$
\Gamma\left(\mathrm{Sp}(-), \mathcal{O}_{\mathrm{Sp}(-)}\right): \text { Ring } \rightarrow \text { Ring }
$$

coincides with the composite $\Gamma \circ F$ : Ring $\rightarrow$ Ring. Thus $\eta$ is a natural transformation $\mathrm{id}_{\text {Ring }} \rightarrow \Gamma \circ F=\Gamma\left(\operatorname{Sp}(-), \mathcal{O}_{\mathrm{Sp}(-)}\right)$. The assumptions on $F$ and $\eta$ guarantee that the functor Sp and natural transformation $\eta$ satisfy Hypothesis 4.3.

Fix a ring $k$ and integer $n \geq 2$. Theorem 4.5 implies that $\Gamma\left(\operatorname{Sp}\left(\mathbb{M}_{n}(k)\right), \mathcal{O}_{\operatorname{Sp}\left(\mathbb{M}_{n}(k)\right)}\right)=0$. By the construction of $G$ and $\mathrm{Sp}=G \circ F$, one readily sees that this equation means that the ringed category $F\left(\mathbb{M}_{n}(k)\right)=\left(\mathcal{X}, \mathcal{O}_{\mathcal{X}}\right)$ has ring of global sections $\Gamma\left(\mathcal{X}, \mathcal{O}_{\mathcal{X}}\right)=0$. It follows from Lemma 4.7 that $\mathcal{O}_{\mathcal{X}}$ is the zero ring object of $\mathcal{X}$.

A similar obstruction, for contravariant functors from the category of rings to the category of ringed toposes, was presented by van den Berg and Heunen in [2, Corollary 6.3]. To compare Theorem 1.2 with their result, we briefly review the necessary terminology.

A topos can be defined tersely as a category with finite limits and power objects. (This is sometimes called an elementary topos, to distinguish from the more specialized notion of a Grothendieck topos.) The quintessential example of a topos is the category of sheaves of sets on a fixed topological space. We will not recall much of topos theory, but the reader is referred to $[8]$ for an in-depth treatment.

If $\mathcal{X}$ and $\mathcal{Y}$ are toposes, a geometric morphism $f: \mathcal{X} \rightarrow \mathcal{Y}$ is a pair $f=\left(f^{*}, f_{*}\right)$ such that $f^{*}: \mathcal{Y} \rightarrow \mathcal{X}$ is and $f_{*}: \mathcal{X} \rightarrow \mathcal{Y}$ are functors (respectively called the inverse image and direct image of $f$ ) such that $\left(f^{*}, f_{*}\right)$ is an adjoint pair and $f^{*}$ preserves finite limits. Let Topos denote the category whose objects are toposes and whose morphisms are geometric morphisms. Given a morphism $f: \mathcal{X} \rightarrow \mathcal{Y}$ in Topos, the right adjoint functor $f_{*}: \mathcal{X} \rightarrow \mathcal{Y}$ preserves limits, including finite products. We claim that $f_{*}$ preserves global sections as well. Indeed, because $f^{*}$ preserves finite limits and a terminal object is the limit of the empty diagram, we have $f^{*}\left(1_{\mathcal{Y}}\right)=1_{\mathcal{X}}$. So

$$
\Gamma(\mathcal{X}, A)=\operatorname{Hom}_{\mathcal{X}}\left(1_{\mathcal{X}}, A\right)=\operatorname{Hom}_{\mathcal{X}}\left(f^{*}\left(1_{\mathcal{Y}}\right), A\right) \cong \operatorname{Hom}_{\mathcal{Y}}\left(1_{\mathcal{Y}}, f_{*}(A)\right)=\Gamma\left(\mathcal{Y}, f_{*}(A)\right) .
$$

This establishes the existence of a "forgetful" functor Topos $\rightarrow$ fpCat that acts trivially on objects and sends a geometric morphism to its direct image part.

A ringed topos is a pair $(\mathcal{X}, \mathcal{O})$ where $\mathcal{X}$ is a topos and $\mathcal{O}$ is a ring object in $\mathcal{X}$. A morphism of ringed toposes $\left(\mathcal{X}, \mathcal{O}_{\mathcal{X}}\right) \rightarrow\left(\mathcal{Y}, \mathcal{O}_{\mathcal{Y}}\right)$ consists of a pair $(f, f)$ where $f=\left(f^{*}, f_{*}\right): \mathcal{X} \rightarrow \mathcal{Y}$ is a geometric morphism and $f: \mathcal{O}_{\mathcal{Y}} \rightarrow f_{*} \mathcal{O}_{\mathcal{X}}$ is a morphism of ring objects in $\mathcal{Y}$. The category RingedTopos is the category of ringed toposes and their morphisms. There is a "forgetful" functor RingedTopos $\rightarrow$ RingedCat, which acts trivially on objects and acts on morphisms 
by $(f, \underline{f}) \mapsto\left(f_{*}, f\right)$. Clearly this functor preserves global sections and reflects which ringed toposes have trivial structure ring objects. It is now quite clear that Theorem 1.2 still holds when the category RingedCat is replaced by the category RingedTopos.

Remark 4.8. The result [2, Corollary 6.3] of van den Berg and Heunen states that any functor $F:$ Ring $^{\mathrm{op}} \rightarrow$ RingedTopos that extends the usual Zariski spectrum must assign the trivial object to $\mathbb{M}_{n}(\mathbb{C})$ for $n \geq 3$. Their conclusion is much stronger than the claim that $\mathbb{M}_{n}(\mathbb{C})$ is assigned a ringed topos with trivial structure sheaf, and actually stems from a topological obstruction about functors to the category of toposes [2, Corollary 6.2]. Their result also relies crucially on the Kochen-Specker Theorem [6] (as did the results of [10]).

By contrast, Theorem 1.2 (and its ringed topos version) is of a more algebro-geometric nature: while the topos (a topological structure) assigned to $\mathbb{M}_{n}(k)$ may not be trivial, the corresponding sheaf of rings (which enhances the topological structure to a geometric structure) must be zero. Furthermore, because the theorem is valid for $n=2$, it is clearly independent of the Kochen-Specker Theorem.

We conclude with a question that is suggested by Theorem 1.2 and Remark 4.8. Let Cat denote the category of categories with functors for morphisms. (Recall Remark 4.1 regarding size issues.) We have already defined a forgetful functor Topos $\rightarrow \mathrm{fpCat}$ sending geometric morphisms to their direct image parts. Composing further with the forgetful functor $\mathrm{fpCat} \rightarrow$ Cat yields a forgetful functor Topos $\rightarrow$ Cat. Taking inverse images of geometric morphisms $\left(f \mapsto f^{*}\right)$ provides another forgetful functor Topos ${ }^{\text {op }} \rightarrow$ Cat. By the trivial category we mean the terminal object in Cat (and fpCat), the category with one object and one morphism.

We know that any functor Ring ${ }^{\text {op }} \rightarrow$ RingedTopos extending the Zariski spectrum must assign the trivial object to $\mathbb{M}_{3}(\mathbb{C})$ by van den Berg and Heunen's result. Even if one tries to construct a functor Ring ${ }^{\text {op }} \rightarrow$ RingedCat extending the spectrum but generalizing the underlying topos, Theorem 1.2 shows that the ring object assigned to $\mathbb{M}_{3}(\mathbb{C})$ must be trivial. Since van den Berg and Heunen's obstruction stems from an underlying topological (KochenSpecker) obstruction, we ask whether the same kind of underlying obstruction occurs in the case of RingedCat.

Question 4.9. Fix an integer $n \geq 3$. Let $F:$ Ring $^{\text {op }} \rightarrow \mathrm{fpCat}$ be a functor whose restriction to cRing $^{\text {op }}$ is the composite of Spec: cRing $^{\mathrm{op}} \rightarrow$ Topos with the forgetful functor Topos $\rightarrow$ fpCat. Does it follow that $F\left(\mathbb{M}_{n}(\mathbb{C})\right)$ is the trivial category?

More generally, suppose that $F:$ Ring $^{\text {op }} \rightarrow$ Cat (respectively, $F:$ Ring $\rightarrow$ Cat) is a functor whose restriction to $\mathrm{cRing}$ is the composite of Spec: $\mathrm{cRing}^{\mathrm{op}} \rightarrow$ Topos with the forgetful functor Topos $\rightarrow$ Cat (respectively, Topos ${ }^{\text {op }} \rightarrow$ Cat). What can be said about $F\left(\mathbb{M}_{n}(\mathbb{C})\right)$ ?

\section{REFERENCES}

1. Benno van den Berg and Chris Heunen, No-go theorems for functorial localic spectra of noncommutative rings, Proceedings 8th International Workshop on Quantum Physics and Logic, Nijmegen, Netherlands, October 27-29, 2011 (Bart Jacobs, Peter Selinger, and Bas Spitters, eds.), Electronic Proceedings in Theoretical Computer Science, vol. 95, Open Publishing Association, 2012, pp. 21-25. 
2. __ Extending obstructions to noncommutative functorial spectra, available online at http://www.cs.ox.ac.uk/people/chris.heunen/publications/2012/nogo/nogo.pdf, preprint, 2012 .

3. George M. Bergman, An invitation to general algebra and universal constructions, Henry Helson, Berkeley, CA, 1998, updated continuously at http://math.berkeley.edu/ gbergman/245/index.html MR 1650275 (99h:18001)

4. Robin Hartshorne, Algebraic geometry, Springer-Verlag, New York, 1977, Graduate Texts in Mathematics, No. 52. MR 0463157 (57 \#3116)

5. Peter T. Johnstone, Sketches of an elephant: a topos theory compendium. Vol. 2, Oxford Logic Guides, vol. 44, The Clarendon Press Oxford University Press, Oxford, 2002. MR 2063092 (2005g:18007)

6. Simon Kochen and E. P. Specker, The problem of hidden variables in quantum mechanics, J. Math. Mech. 17 (1967), 59-87. MR 0219280 (36 \#2363)

7. Maxim Kontsevich and Alexander Rosenberg, Noncommutative stacks, MPMI preprint number 2004-37, available online at http://www.mpim-bonn.mpg.de/preprints, 2004.

8. Saunders Mac Lane and Ieke Moerdijk, Sheaves in Geometry and Logic: A first introduction to topos theory, Universitext, Springer-Verlag, New York, 1994, Corrected reprint of the 1992 edition. MR 1300636 (96c:03119)

9. D. O. Orlov, Quasicoherent sheaves in commutative and noncommutative geometry, Izv. Ross. Akad. Nauk Ser. Mat. 67 (2003), no. 3, 119-138. MR 1992195 (2004m:14026)

10. Manuel L. Reyes, Obstructing extensions of the functor Spec to noncommutative rings, Israel J. Math. 192 (2012), no. 2, 667-698. MR 3009738

11. Angelo Vistoli, Grothendieck topologies, fibered categories and descent theory, Fundamental Algebraic Geometry: Grothendieck's FGA Explained, Math. Surveys Monogr., vol. 123, Amer. Math. Soc., Providence, RI, 2005, pp. 1-104. MR 2223406

Department of Mathematics, Bowdoin College, 8600 College Station, Brunswick, ME 04011-8486

E-mail address: reyes@bowdoin.edu

$U R L:$ http: //www. bowdoin.edu/〜reyes / 